University of New Hampshire

University of New Hampshire Scholars' Repository

Center for Coastal and Ocean Mapping

Center for Coastal and Ocean Mapping

7-1991

\title{
Angular dependence of 12-kHz seafloor acoustic backscatter
}

Christian de Moustier

University of California - San Diego

Dimitri Alexandrou

Duke University

Follow this and additional works at: https://scholars.unh.edu/ccom

Part of the Oceanography and Atmospheric Sciences and Meteorology Commons

\section{Recommended Citation}

C. de Moustier and D. Alexandrou, 'Angular dependence of 12-kHz seafloor acoustic backscatter', The Journal of the Acoustical Society of America, vol. 90, no. 1, pp. 522-531, 1991.

This Journal Article is brought to you for free and open access by the Center for Coastal and Ocean Mapping at University of New Hampshire Scholars' Repository. It has been accepted for inclusion in Center for Coastal and Ocean Mapping by an authorized administrator of University of New Hampshire Scholars' Repository. For more information, please contact Scholarly.Communication@unh.edu. 


\title{
Angular dependence of 12-kHz seafloor acoustic backscatter
}

\author{
C. de Moustier \\ Marine Physical Laboratory, Scripps Institution of Oceanography, University of California, San Diego, \\ 9500 Gilman Drive, La Jolla, California 92093-0205 \\ D. Alexandrou \\ Department of Electrical Engineering, Duke University, Durham, North Carolina 27706
}

(Received 4 October 1990; accepted for publication 13 March 1991)

\begin{abstract}
The angular dependence of seafloor acoustic backscatter, measured with a $12-\mathrm{kHz}$ multi narrow-beam echo-sounder at two sites in the central North Pacific with water depths of 1500 and $3100 \mathrm{~m}$, respectively, has been determined for incidence angles between $0^{\circ}$ and $20^{\circ}$. The acoustic data consist of quadrature samples of the beamformed echoes received on each of the $162.66^{\circ}$ beams of a Sea Beam echo-sounder. These data are subjected to adaptive noise cancelling for sidelobe interference rejection, and the centroid of each echo is determined. After corrections for the ship's roll and raybending effects through the water column, the angles of arrival are converted to angles of incidence by taking athwartships apparent bottom slopes into account. For each beam, the mean echo power received is normalized by the corresponding insonified area that depends on the transmit and receive beam patterns, the ship's roll angle and the local bottom slope. For lack of system calibration, the data are presented as relative mean energy levels in $1^{\circ}$ bins. Comparison of these results with theoretical angular dependence functions, based on the Helmholtz-Kirchhoff model for backscatter from a rough surface, indicates that a good fit is obtained in the angular sector from $5^{\circ}$ to $20^{\circ}$ incidence. In the near-nadir sector $\left(0^{\circ}\right.$ to $\left.5^{\circ}\right)$, the data suffer from high variance making the estimate unreliable. The data processing methods presented constitute one of the elements necessary to compile a map of seafloor acoustic backscatter from acoustic measurements made with a multinarrow beam echo-sounder. The angular dependence function obtained will ultimately be used to normalize the backscatter measurements in the athwartships direction.
\end{abstract}

PACS numbers: 43.20.Fn, 43.30.Gv, 43.30.Hw

\section{INTRODUCTION}

In the larger context of remote classification of the deep seafloor by high-frequency acoustics methods (10's to 100 's of $\mathrm{kHz}$ ), one may ask whether bottom types can be differentiated on the basis of the angular dependence of the acoustic energy they backscatter. Also, what are the parameters that control this angular dependence? Is surface roughness mostly responsible for the backscattered signal levels observed or does volume scattering within the sediments play an important role?

For logistics reasons, previous work on the subject has been done mostly in shallow water, ${ }^{1-9}$ and the corresponding results provide valuable references for work in the deep ocean. Compared to coastal environments, where bottom properties can change on length scales of tens of meters or less, the deep ocean floor can be expected to appear relatively uniform on length scales of kilometers. However, outcrops and changes in lithology have also been found to occur in the deep ocean on scales a few hundred meters, ${ }^{10}$ thus presenting a particular challenge to the task of remote sensing of seafloor characteristics. Assuming one can produce reliable generic curves of backscattering strength as a function of angle of incidence for various bottom types with uniform composition, departures from the generic curves in mea- sured angular dependence functions could provide clues to changing substrate or relief characteristics.

As was shown in a previous paper by de Moustier, ${ }^{11}$ one of the tools that has potential for such applications is the multi narrow-beam echo-sounder that has been used extensively over the past 10 years to map the bottom of the ocean with swaths of depth samples co-registered across the ship's track. The multi narrow-beam geometry is particularly well suited to the task of deriving an angular dependence of seafloor acoustic backscatter because it provides both the high angular resolution needed for such measurements and quantitative estimates of apparent bottom slopes for each measurement cycle. The present development shows that the bathymetric information is a crucial part of the problem.

The purpose of this paper is to present results derived from seafloor acoustic backscatter measurements made with a 12-kHz, 16-beam, Sea Beam echo-sounder. Starting with digitized and tape-recorded quadrature samples of the seafloor echoes received on each preformed beam, a number of processing steps have been developed to arrive at an angular dependence function of seafloor acoustic backscattering strength. For lack of system calibration, these results are presented as relative levels, normalized to their value at vertical incidence. For verification purposes, these angular dependence functions are compared with normalized scatter- 
ing strength curves obtained by modeling the surface scattering as a sum of two components: one due to scattering at the water sediment interface, and the other due to sediment volume scattering. For this we follow the development presented by Jackson et al. ${ }^{6}$ and a brief review of the corresponding theory is given in Sec. I. Details of the data processing procedures implemented, and their validation through computer simulations using the REVerberation GENerator software package (REVGEN), ${ }^{12}$ are given in Sec. II. In Sec. III, the results and their comparison with theory are discussed based on both geometric and physical arguments. The implication for compiling maps of seafloor acoustic backscatter measured with multi narrow-beam echosounders, for bottom differentiation, and for signal conditioning and processing in bathymetric applications are also considered.

\section{THEORY}

A conventional definition of the received level backscattered from an area $A$ (e.g., Urick $^{13}$ ) is

$$
\begin{aligned}
& 10 \log _{10}\left[I_{s}(\theta)\right] \\
& \quad=10 \log _{10}\left(I_{0} \int_{A} s(\phi) b(\phi, \psi) b^{\prime}(\phi, \psi) r^{-4} 10^{-2 \alpha_{V} / 10} d A\right),
\end{aligned}
$$

where $I_{s}$ is the scattered intensity at the receiver, $I_{0}$ is the incident intensity taken on axis at unit distance from the source, $b(\phi, \psi)$ and $b^{\prime}(\phi, \psi)$ are, respectively, the source and receiver beam pattern functions, $r$ is the range from the source to the elemental scattering surface area $d A$ and $\alpha_{v}$ is the seawater absorption coefficient. $10 \log _{10}[s(\phi)]$ is the backscattering strength per unit area.

In practice, $I_{s}(\theta)$ is an average of instantaneous backscatter components, $I_{s}(\phi)$, integrated over the elemental area $d A$, so that the angle $\theta$ can be viewed as the angle of incidence obtained by averaging incremental values of $\phi$ over the area insonified by the beam patterns. Provided instantaneous samples of the backscattering process are available, it is possible to envisage normalizing these samples by the corresponding beam pattern coefficients and incremental area. This is the basis of the normalization scheme we have developed for the Sea Beam acoustic data presented here. The end result is a backscattering strength:

$S(\theta)=10 \log _{10}\left(I_{0}^{-1} \int_{A} \frac{I_{s}(\phi) r^{4} 10^{2 \alpha_{r} / 10}}{b(\phi, \psi) b^{\prime}(\phi, \psi)} d A\right)$.

Given that we are only concerned here with measurements over an angular sector spanning about $20^{\circ}$ from vertical incidence, we shall adapt to the Sea Beam case Jackson et al. ${ }^{6}$ model of bottom backscattering strength, expressed as a sum of components scattered at the sediment water interface and throughout the sediment volume. We use the relevant equations from their paper [Eqs. (21), (38), (39), $(41)-(43)]$ that were derived in terms of the grazing angle $\theta_{\mathrm{g}}=(\pi / 2)-\theta$.

The interface scattering term is derived under the Kirchhoff approximation, requiring that the radius of curvature of the surface insonified be large compared to the acoustic wavelength. For Sea Beam with an acoustic frequency of
$12.158 \mathrm{kHz}$, this is equivalent to requiring that the radii of curvature of the surface be greater than $0.04 \mathrm{~m}$. This condition is easily satisfied in the data presented here because we are dealing with deep sea sediment fields.

The contribution to the overall surface scattering strength that is due to scattering at the water sediment interface is given by

$\sigma\left(\theta_{g}\right)=\frac{g^{2}(\pi / 2)}{8 \pi \sin ^{2}\left(\theta_{g}\right) \cos ^{2}\left(\theta_{g}\right)} \int_{0}^{\infty} \exp \left(-q u^{2 \alpha}\right) J_{0}(u) u d u$,

with

$$
q=\sin ^{2}\left(\theta_{g}\right) \cos ^{-2 \alpha}\left(\theta_{g}\right) C_{h}{ }^{2} 2^{1-2 \alpha} k_{a}^{2(1-\alpha)},
$$

and the plane-wave reflection coefficient at normal incidence,

$$
g(\pi / 2)=(\rho v-1) /(\rho v+1),
$$

where $v$ is the ratio of the sediment compressional wave speed over the sound speed in the overlying water and $\rho$ is the ratio of mass densities of sediment over water. $J_{0}$ is the zeroth-order Bessel function of the first kind, $k_{a}$ is the acoustic wave number, and $\alpha$ is related to the roughness spectrum of the interface. The model assumes isotropic Gaussian statistics for the interface roughness, with a power law spectrum expressed in terms of the wave number $k$ and the roughness parameter $\beta$ according to

$$
W(k)=\beta k^{-r},
$$

and $\alpha=(\gamma / 2)-1,(0<\alpha<1)$. For high-frequency bottom backscatter, $3 \leqslant \gamma \leqslant 3.5$, so that $0.5 \leqslant \alpha \leqslant 0.75$. Related to this power spectrum is a structure function $D(r)$, which depends only on the horizontal distance $r$ between elements of the surface and the wave number $k$ :

$$
D(r)=C_{h}{ }^{2}{ }^{2 \alpha} \text {, }
$$

where $C_{h}$ depends only on $\alpha$ and $\beta$ :

$$
C_{h}^{2}=\left[2 \pi \beta \Gamma(2-\alpha) 2^{-2 \alpha}\right] /[\alpha(1-\alpha) \Gamma(1+\alpha)] .
$$

Note that in Jackson et al., ${ }^{6} C_{h}$ is dimensioned in units of $\mathrm{cm}^{(1-\alpha)}$, so that comparison of their results to results using length scales based on meters requires that the corresponding values of $C_{h}$ be multiplied by $10^{-2(1-\alpha)}$.

At vertical incidence, $\left(\theta_{g}=90^{\circ}\right)$, Eq. (3) is indeterminate but a closed-form solution is found by making the change of variables $w=q u^{2}$ and integrating to get,

$$
\sigma\left(\frac{\pi}{2}\right)=g^{2}\left(\frac{\pi}{2}\right)(8 \pi \alpha)^{-1} C_{h}^{-2 / \alpha}\left(2 k_{a}^{2}\right)^{(\alpha-1) / \alpha} \Gamma\left(\frac{1}{\alpha}\right) .
$$

The scattering strength $10 \log _{10}(\sigma)$ for the interface contribution is plotted in Fig. 1 at the Sea Beam acoustic frequency $(12.158 \mathrm{kHz})$ for various values of the parameter $\alpha$ and with a value for $C_{h}$ set at $10^{(2 \alpha-3)}$, corresponding to Jackson's $C_{h}=0.1$.

The contribution to the surface scattering strength due to sediment volume scattering is a variant of the composite 


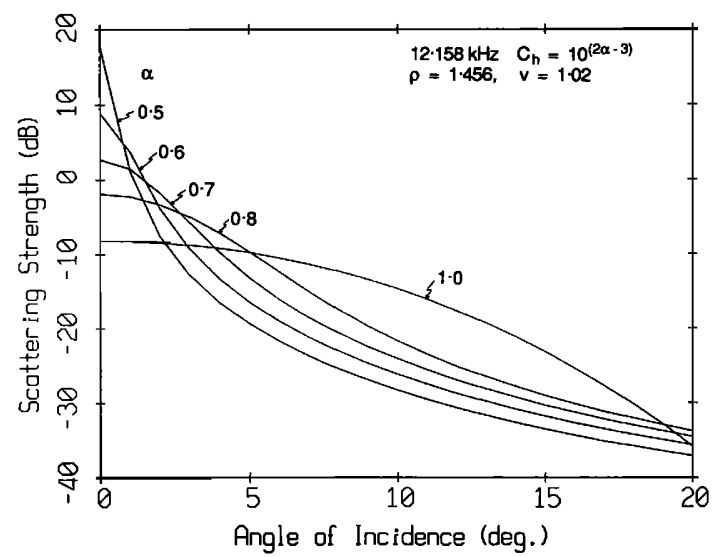

FIG. 1. Scattering strength versus angle of incidence computed from Eqs. (3) -(9) for $12.158 \mathrm{kHz}$ and values of $\alpha$ between 0.5 and 1 . The parameter $C_{h}$ was set at $10^{2 a-3}$, corresponding to Jackson's ${ }^{6} C_{h}=0.1$ case.

roughness model at large grazing angle that averages smallscale contributions over the large scale bottom slopes $s_{x}$ with rms slope $s$ :

$$
\sigma\left(\theta_{g}\right)=\left(\pi^{1 / 2} s\right)^{-1} \int_{-\theta_{g}}^{\infty} \sigma_{v s}\left(\theta_{g}+s_{x}\right) \exp \left(-s_{x}^{2} s^{-2}\right) d s_{x},
$$

with the sediment volume scattering cross section,

$$
\sigma_{v s}\left(\theta_{g}\right)=\frac{5 \sigma_{v}\left[1-g^{2}\left(\theta_{g}\right)\right]^{2} \sin ^{2}\left(\theta_{g}\right)}{\alpha_{b} \ln 10 \sin \left(\theta_{b}\right)},
$$

and the plane-wave reflection coefficient

$$
g\left(\theta_{g}\right)=\frac{\rho v \sin \left(\theta_{g}\right)-\sin \left(\theta_{b}\right)}{\rho v \sin \left(\theta_{g}\right)+\sin \left(\theta_{b}\right)},
$$

where the refracted angle $\theta_{b}$ follows Snell's law:

$$
\sin \left(\theta_{b}\right)=\left\{1-\left[v \cos \left(\theta_{g}\right)\right]^{2}\right\}^{1 / 2} .
$$

In Eq. (11), $\alpha_{b}$ is the sediment attenuation coefficient for compressional waves and the subscript $s$ designates small scale in accordance with Jackson's notation. This is a modified version of Jackson's Eq. (21) that included a shadowing term that does not come into play in the angular sector of interest here, particularly since the rms slope $s$ is assumed to be small $(<0.1)$. Likewise, for the application discussed here, the integrand need only be summed over a range of slopes of $\pm 10^{\circ}$, beyond which its contributions to the integral are negligible. The underlying assumptions of this model are the absence of multiple scattering which translates into $\left(\sigma_{v} / \alpha_{b}\right)<0.004$.

The overall surface scattering strength is then obtained by summing both contributions defined by Eqs. (3) and (10). In the following, we shall use the ratio $\sigma_{v} / \alpha_{b}$ and the parameters $\alpha$ and $\beta$ to fit theoretical curves to the measurements presented here.

\section{DATA PROCESSING METHODOLOGY}

The seafloor acoustic backscatter data presented here were recorded from Sea Beam echo-sounders, installed aboard research vessels (R/V's) T. WASHINGTON and ATLANTIS II, with a special purpose data acquisition system designed at the Marine Physical Laboratory (MPL) ${ }^{14}$ This system buffers the $12.158-\mathrm{kHz}$ analog signals at the output of the Sea Beam beamformer and after basebanding, quadrature sampling and analog-to-digital conversion, the signals are recorded on a 9-track magnetic tape as well as displayed in real-time on a video monitor. The ship's roll information is also digitized and recorded at the same time. Therefore, for each ping the data consist of 33 channels simultaneously sampled at about $1 \mathrm{kHz}$ per channel, that is 16 complex channels with the in-phase $(I)$ and quadrature $(Q)$ components of the returns plus the ship's roll. A real-time bottom tracking algorithm sets the start of the data digitization window 100-200 ms ahead of the first bottom return, and depending on water depth, the window can be as wide as $1.4 \mathrm{~s}$ to account for increased travel times for returns received on the beams farthest from vertical incidence.

In this paper, we concern ourselves with processing these data to derive an angular dependence function of seafloor acoustic backscatter at $12 \mathrm{kHz}$ in the angular sector sampled by the Sea Beam system: roughly $\pm 20^{\circ}$ about vertical incidence. When the ship rolls, this angular sector can extend beyond $30^{\circ}$ incidence, but the angular sampling performed in this fashion is usually too sparse for our needs, so we restrict ourselves to $\pm 20^{\circ}$ to insure relatively uniform sample counts in bins $1^{\circ}$ wide.

The processing methods described below result from a close coupling between tests on the recorded acoustic data and tests on well-controlled simulated data obtained through the REVGEN simulation software package. Iterations between the two types of data, recorded and simulated, allowed us to refine the algorithms and verify the potential sources of errors. We found that three main processing steps were required to obtain an angular dependence function with these acoustic data: (1) sidelobe interference removal, (2) angular corrections (roll, refraction, and athwartships bottom slopes) and (3) geometric corrections (beam pattern and area insonified).

\section{A. Sidelobe interference removal}

Inherent in the multibeam geometry is the fact that each preformed beam has sidelobes pointing within the main beam of all the other beams. As a result, a strong echo received in the mainlobe of one of the beams will also be seen by all the other beams through their sidelobes pointing in that direction. This situation is particularly damaging in the near-specular region where sidelobe contributed returns and bottom echoes tend to overlap. However, this problem can be alleviated using adaptive noise cancelling techniques provided the full waveform is available. To this end, we apply a joint least-squares lattice algorithm to the 16 complex acoustic data channels, using the channel with the strongest return (usually a specular beam) as the reference and performing the adaptive cancellation on the 15 other channels. The cor- 
responding algorithm and its performance are described in detail by Alexandrou and de Moustier, ${ }^{15}$ and it will not be repeated here. However, two modifications were made to this original processing scheme: (1) The likelihood parameter of the filter is monitored to determine time intervals over which the filtered output is accepted, and (2) the beams directly adjacent to the reference channel are not subject to sidelobe cancellation.

The first modification is based on the fact that the likelihood parameter quickly approaches unity when the sidelobe interference sets in, indicating a strong correlation between the reference and the primary channels. This time window is currently set to correspond to a drop of $-20 \mathrm{~dB}$ on either side of the peak value of the likelihood parameter. This was done to prevent the filter from introducing noise at the output, as would be the case when a weak correlation, or no correlation exist between the reference and the primary channels.

The second modification deals with the fact that the mainlobes of adjacent beams intersect near their $-3 \mathrm{~dB}$ down point (Fig. 2), and cross talk between them is therefore inevitable. Because the sidelobe filtering scheme works on correlated elements between two channels, a side effect of this sidelobe cancellation is that the reference channel can cancel some of the main return in adjacent beams. Depending on the sidelobe level present in the data on a given ping, it is often necessary to run three passes of the filter, starting with the specular return as the reference channel, and then using the two adjacent beams as reference in pass two and three, respectively. In each case, the beams directly adjacent to the reference beam are not processed through the filter during that pass.

Effective cancellation of the sidelobe interference is an important part of the processing as it not only affects the amount of energy recorded versus actually backscattered by the seafloor in a given direction, but it also affects the ability to determine the time of arrival of the seafloor echo on each beam which is needed in subsequent processing steps.

\section{B. Time of arrival and mean energy of the return for each beam}

Having isolated the bottom return from other interfering signals in each beam, it is a relatively simple matter to determine the time of arrival of the return and compute its mean energy. The time of arrival is determined via a two step process: (1) using the magnitude square $\left(I^{2}+Q^{2}\right)$ of the signals received on each beam, a first guess at the time of arrival is achieved by finding the peak value of the corresponding low-pass filtered time series, using a simple running mean of 100 samples; and (2) refining this initial estimate by finding the median of the samples with squared magnitude greater than the mean noise power on that same beam, and contained within a window at most $400 \mathrm{~ms}$ wide and centered on the initially estimated time sample. The mean energy in the return is then simply the averaged sum of the magnitude square samples exceeding the mean noise power and contained within the same window. As will be shown later, this mean energy calculation must be refined by taking into account the beam pattern effects.

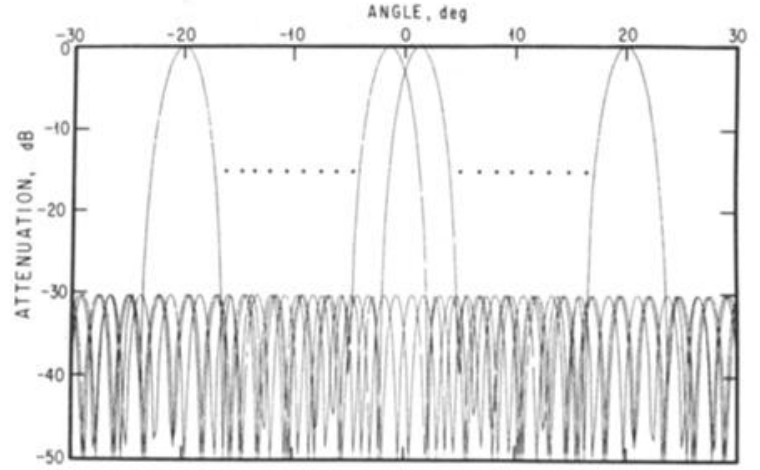

FIG. 2. Theoretical receive beam patterns for the Sea Beam multibeam echo-sounder (from de Moustier ${ }^{11}$ ). Beams are roughly $2.66^{\circ}$ wide and are spaced on $2.66^{\circ}$ centers between $\pm 20^{\circ}$ about the ship's vertical center line. Theoretical 30-dB sidelobe reduction is achieved through Dolph-Tchebychev shading. Only 4 out of the 16 beams are plotted for clarity of the picture.

The depth and horizontal distances corresponding to the time of arrival of the seafloor returns on each beam are then computed by simple geometry once the corresponding effective angles of arrival have been established.

\section{Roll and refraction corrections to determine the angle of arrival}

The Sea Beam system's narrow beam echo-sounder preforms 16 receive beams whose directions are fixed within the ship's reference frame. These beams are spaced $2.66^{\circ}$ apart and span $\pm 20^{\circ}$ on either side of the ship's vertical center line. As a result, there is no beam aligned with the ship's vertical center line, instead the two centermost beams are $1.33^{\circ}$ on either side of this line. The ship's roll is digitized and recorded concurrently with the complex acoustic data in the data acquisition system. This roll data is used to translate the measurements from the ship's reference frame to a true vertical reference. Because the difference in time of arrival between the first echoes received on near-specular beams and the beams farthest from vertical incidence can easily exceed $1 \mathrm{~s}$ at average ocean depths of $4 \mathrm{~km}$, and because the ship's roll period is typically a few seconds (e.g., $5 \mathrm{~s}$ for $\mathrm{R} / \mathrm{V}$ T. WASHINGTON), it is necessary to assign a different roll value for each individual beam. This is done by computing the average roll angle in a time window roughly $200 \mathrm{~ms}$ wide and centered at the estimated time of arrival of the bottom return for each beam.

An additional angular correction is required to determine the effective angle of arrival of the returns received on each beam, as refraction effects through the water column and at the face of the hydrophone array must be taken into account. The processing scheme we use for these corrections is similar to that used by the Sea Beam system's echo processor: first the refraction at the face of the array is computed by simple application of Snell's law, converting the nominal beam directions $\theta_{n}(i)$ to angles of arrival $\theta_{a}(i)$ in the ship's reference frame:

$$
\theta_{a}(i)=\arcsin \left\{\left(C_{s} / C_{0}\right) \sin \left[\theta_{n}(i)\right]\right\},
$$

where $C_{s}$ is the sound speed at the face of the array, a value 
inferred from the temperature profile obtained during an XBT cast (or measured directly and continuously in more recent multibeam echo-sounders, ${ }^{16}$ ) and $C_{0}$ is the array design sound speed. For the Sea Beam system,

$$
\theta_{n}(i)=-20+(i-1)(8 / 3), \quad i=1, ?{ }^{7} 16 .
$$

The effective angles of arrival $\theta_{e}(i)$ are then obtained by adding the ship's roll angle to the $\theta_{a}(i)$ 's and applying Snell's law to account for refraction through the water column:

$\theta_{e}(i)=\arcsin \left\{\left(C_{a} / C_{s}\right) \sin \left[\theta_{a}(i)+\operatorname{roll}(i)\right]\right\}, \quad i=1,16$,

where $C_{a}$ is the harmonic sound speed computed from the face of the hydrophone array to the depth corresponding to the first bottom return in the ping.

To determine depth and horizontal distances from these effective angles of arrival and the corresponding time of arrival of the echoes on each beam, a straight propagation path is assumed, with $C_{a}$ as the sound speed. The validity of this assumption was checked by computing eigenrays from the array to the bottom, over the angular sector of interest, using measured sound velocity profiles as input to the conjugate gradient ray tracing model of the Generic Sonar Model. Straight path calculations with the harmonic sound speed, averaged over the water column, yielded travel times that were within less than $0.1 \mathrm{~ms}$ of the travel times of the corresponding eigenrays. The straight paths can therefore be considered reliable estimates of the slant range to the bottom. Note that in practice, depths are usually reported in uncorrected meters, i.e., referenced to a constant sound speed of $1500 \mathrm{~m} / \mathrm{s}$. The horizontal distances are usually true distances calculated with the harmonic sound speed.

\section{Bottom slope correction}

If we bin the data after the processing steps described above (sidelobe cancellation, roll and refraction correction and energy calculations) and, considering a few pings, plot the average value of the energy in each bin as a function of angle of arrival, the result is usually not symmetric with respect to vertical $\left(0^{\circ}\right)$ [Fig. 3(a) ]. This asymmetry is due to the fact that the bottom is rarely a flat horizontal plane. Because the measurements made with a multi narrow-beam echo-sounder are highly directional, it is important to take bottom slopes into account when deriving an angular dependence function of seafloor acoustic backscatter. With beams spaced roughly $2.66^{\circ}$ apart, the average horizontal interval between depth samples measured by the Sea Beam system in the athwartships direction is about $5 \%$ of the water depth below the ship, and athwartships slopes calculated with these depth samples are apparent slopes.

Taking the apparent athwartships slopes into account, the angles of arrival are converted to angles of incidence and the angular dependence function becomes symmetric with respect to vertical [Fig. 3(b)]. To compute the apparent athwartships bottom slopes, we fit the set of depth and horizontal distance pairs obtained for each ping with a piecewise continuous chain of straight lines (Fig. 4). Each straight line
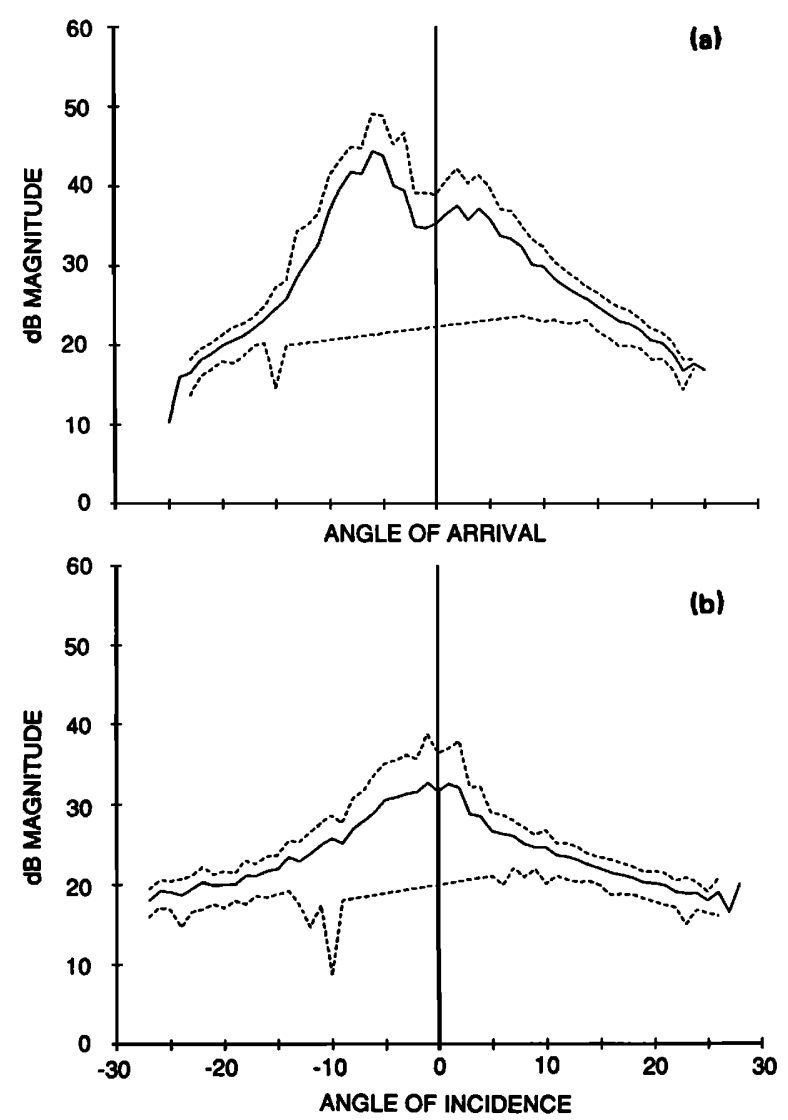

FIG. 3. Effect of athwartships bottom slopes seen in the angular dependence of mean echo power by (a) asymetry of the function about $0^{\circ}$ because, without correction, the angles reflect the direction of arrival of the echoes with respect to vertical incidence, not the angle of incidence with respect to the bottom; (b) same data after corrections for athwartships slopes have been applied. The dashed lines represent 1 s.d. about the mean.

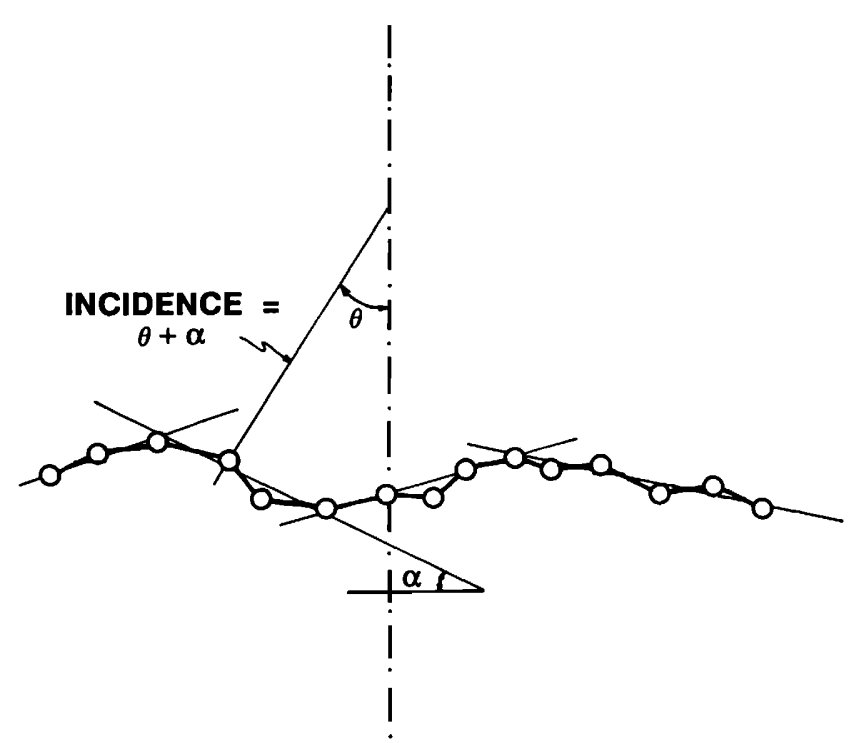

FIG. 4. Conversion between the angle of arrival $\theta$ and the angle of incidence $\theta+\alpha$ where $\alpha$ designates the slope of the straight line segment fit through a subset of the points in the instantaneous cross-track bathymetric profile. 
$z=m x+n$ in the chain is determined by the constrained absolute deviation criterion:

$$
\sum_{i=1}^{N}\left|z_{i}-n-m x_{i}\right|<\text { threshold, }
$$

where $z_{i}$ and $x_{i}$ represent the depth and horizontal distance samples, respectively. Initial values for the slope $m$ and the intercept $\boldsymbol{n}$ are obtained through a standard least-squares fit to a straight line, with standard deviations (s.d.) arbitrarily set to 1 . This method has the advantage of providing some measure of "goodness of fit" through the mean absolute deviation of the $z_{i}$ 's from the fitted line, in spite of the fact that uncertainties associated with the data are unknown. Thus, by varying the threshold parameter in Eq. (17), one can tighten or relax the criterion depending on the fit desired. In addition, because the minimum absolute deviation criterion is less sensitive to outliers than a conventional least-squares method $^{17}$ we found it to be useful when dealing with depth samples from outer beams for which errors are typically greater than their center beams counterparts. The difficulties of estimating the errors associated with each depthhorizontal distance pair is also a reason why segments spanning across several points athwartships are preferred to beam-to-beam slope values. For each ping, the set of apparent athwartships bottom slopes $m$ so determined is converted to angular form and added to the corresponding effective angles of arrival $\theta_{e}(i), i=1,16$ from Eq. (16), yielding estimates of the angle of incidence for the return received on each beam.

\section{E. Beam pattern and insonified area correction}

The footprint of any one beam on the seafloor can be approximated by the intersection of the beam pattern with a plane. For a beam with angular dimensions $2 \psi$ in the foreaft direction and $2 \phi$ in the athwartship direction, this footprint on a horizontal plane will be an ellipse (Fig. 5). For a beam with effective angle of arrival $\theta_{e}$ and a bottom at depth $D$, we define an apparent depth $D_{a}$ with respect to the angle of incidence $\theta_{i}$ as the perpendicular to the plane:

$$
D_{a}=D \cos \left(\theta_{i}\right) / \cos \left(\theta_{e}\right) \text {, }
$$

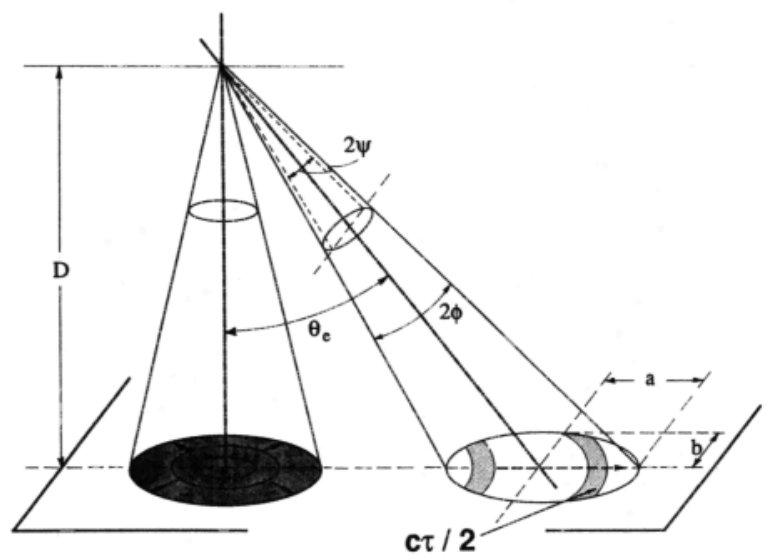

FIG. 5. Geometry of vertical versus outer beams illustrating the beam limitation in the near-nadir region and the pulse limitation in the outer beams. $\tau$ represents the pulse length of incident sound, $c$ is the sound speed. so that the the semi major axis $a$ and semi minor axis $b$ of this ellipse are obtained by

$$
\begin{aligned}
& a=0.5 D_{a}\left(t_{1}-t_{2}\right) \\
& b=a \tan (\psi) /\left(\cos \left(\theta_{i}\right) t_{3}^{1 / 2}\right)
\end{aligned}
$$

where

$$
t_{1}=\tan \left(\theta_{i}+\phi\right), \quad t_{2}=\tan \left(\theta_{i}-\phi\right),
$$

and

$$
t_{3}=\left(t_{1}+t_{2}\right) \tan \left(\theta_{i}\right)-\tan ^{2}\left(\theta_{i}\right)-t_{1} t_{2} .
$$

As the transmitted pulse propagates from the source, it expands in a spherical fashion and is more or less bound by the angular dimensions of the transmit beam pattern. When the corresponding portion of sphere intersects the seafioor, following our plane seafloor assumption, it will first fully insonify an area and then appear to propagate on the plane as an annulus. Consequently, the area insonified by the pulse at any one time is the area contained within the intersection of the beam pattern footprint and the annulus of the pulse (Fig. 5). For time increments on the order of half a pulse length, the insonified area can be represented by the intersection of the beam footprint and the pulse footprint on the plane, according to the relation,

$$
A=2\left(\int_{x_{1}}^{x_{3}} f(x) d x-\int_{x_{1}}^{x_{2}} g_{1}(x) d x+\int_{x_{3}}^{x_{4}} g_{2}(x) d x\right)
$$

For ease of calculations, we assume a circular annulus for the pulse projection on the plane, so that $g_{1}(x)$ and $g_{2}(x)$ are circles centered at the origin and of respective radii $x_{2}$ and $x_{4}$. The function $f(x)$ represents the ellipse of the footprint (Fig. 6). The $x$ coordinates of the intersections between the circles and the ellipse are obtained by

$$
\begin{aligned}
x= & \left(-x_{0} d_{1}+\left[\left(r^{2}-b^{2}\right) d_{2}+d_{1} x_{0}^{2}\right]^{1 / 2}\right) d_{2}^{-1} \\
& \text { with } d_{1}=b^{2} / a^{2} \text { and } d_{2}=1-d_{1},
\end{aligned}
$$

where $a$ and $b$ are the semi major and minor axes of the ellipse centered at $\left(x_{0}, y_{0}\right)$, and $r$ is the radius of the circle.

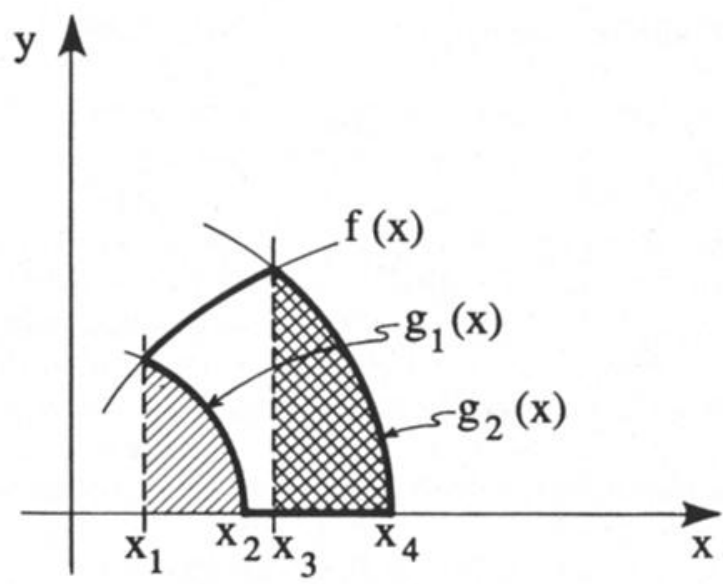

FIG. 6. Area insonified by the pulse length within the footprint of a given beam. $f(x)$ delimits the beam footprint, $g_{1}(x)$ and $g_{2}(x)$ represents, respectively, the trailing and leading edges of the projection of the pulse on a horizontal plane. The bold line encloses half the area of interest that is symmetric with respect to the $x$ axis. 
Assuming a sampling frequency of $1 \mathrm{kHz}$, as in the acoustic data acquisition system we used to acquire Sea Beam acoustic data, the number of samples needed to propagate the pulse through a beam's footprint corresponds to the time span in milliseconds from the moment the pulse first enters the near edge of the footprint until its trailing edge exits the far edge of the footprint (near and far being with respect to vertical incidence). For each of these samples, the area $\boldsymbol{A}$ of Eq. (21) is calculated and a correction factor is computed by

$$
\operatorname{cor}(j)=A(j) b_{m}(j) /\left[R^{4}(j) 10^{0.2 \alpha_{v} R(j)}\right],
$$

where $R(j)$ is the slant range from the center of the hydrophone array to the middle of $A(j)$, and $b_{m}(j)$ is a weighting coefficient that accounts for the varying beam intensity through the footprint. For each beam, these correction factors, Eq. (23), are applied to the individual quadrature samples of the echoes received, and the mean echo power is computed by averaging the sum of the squares of these corrected samples over the number of samples considered.

\section{F. REVGEN computer simulations}

To verify that the correction scheme described above is valid, we ran a number of REVGEN simulations in which the complete geometry of the Sea Beam sonar was used to insonify an ensemble of point scatterers distributed over a plane interface at a prescribed depth, and to receive the corresponding returns. For these simulations, the preformed beams were given fixed directions (no roll) and kept the same $2.66^{\circ}$ beam spacing as the Sea Beam system, but beam No. 1 is aligned with the vertical. Also, to avoid sidelobe interference, the simulation did not include a reflected (coherent) component in the returns. Likewise, no angular dependence was input into the simulation, so that the resulting echoes only account for the distribution of scatterers and the respective beam geometries. The envelopes of these returns, shown in Fig. 7(a), bear close resemblance to those measured with the Sea Beam system in the field (Fig. 8). Likewise, in both cases, the instantaneous correction factors conform quite well with the shape of the echo envelope on each beam.

Assuming the maximum response axis of each preformed beam coincides with the time of arrival of the echo for that beam, the correction factors can be used to constrain reliably the time window over which the average power in the return is calculated. Figs. 7 (a) and 8 seem to support this assumption. So we have developed a routine that detects the peak of the correction function for each beam, presumably corresponding to the maximum response axis of that beam, and the number of samples considered in the average is directly related to the width of the correction function $n \mathrm{~dB}$ down from the peak. In the following we have used $n=-6$ $\mathrm{dB}$ as the threshold, so that reverberation values falling inside this interval are corrected individually and averaged to give an estimate of the mean power in the return.

Comparisons of the average power in the simulated returns with that of the corresponding computed correction factors [Fig. 7(b)] yield good agreement except in the near nadir region where the two curves are seen to diverge with the correction factors exhibiting a steeper slope from vertical
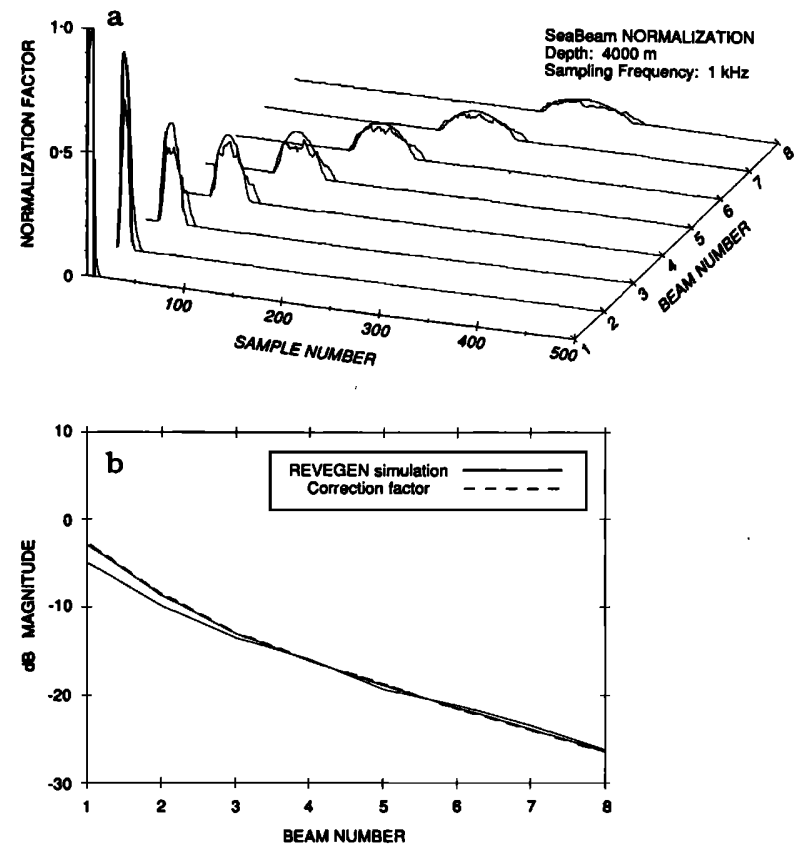

FIG. 7. REVGEN simulation of the Sea Beam backscatter geometry. (a) Simulated returns and their corresponding correction factors. (b) Average power versus beam number (beams are $2.66^{\circ}$ apart, starting at vertical incidence) for a number of simulated pings returns, with the average power in the correction factors overlaid for comparison.

incidence to about $4^{\circ}$ to $5^{\circ}$. We do not have a good explanation for this discrepancy, but as discussed below, this angular sector corresponds to the transition from a beam-limited regime to a pulse-limited regime, and is difficult to control. Examples of the resulting apparent angular dependence functions obtained, before applying the correction factors, for simulated depths of 1000 and $4000 \mathrm{~m}$, respectively, are shown in Fig. 9. The correction yields lines that are essentially horizontal except for a deviation from horizontal near nadir because of the discrepancy mentioned above [Fig. 7(b) ].

When comparing the mean energy values calculated over the same fixed time window centered on the centroid of the return for each beam, as described in Sec. II B, with those calculated within the $6 \mathrm{~dB}$ down points of the correction functions, the former yield proportionately higher relative

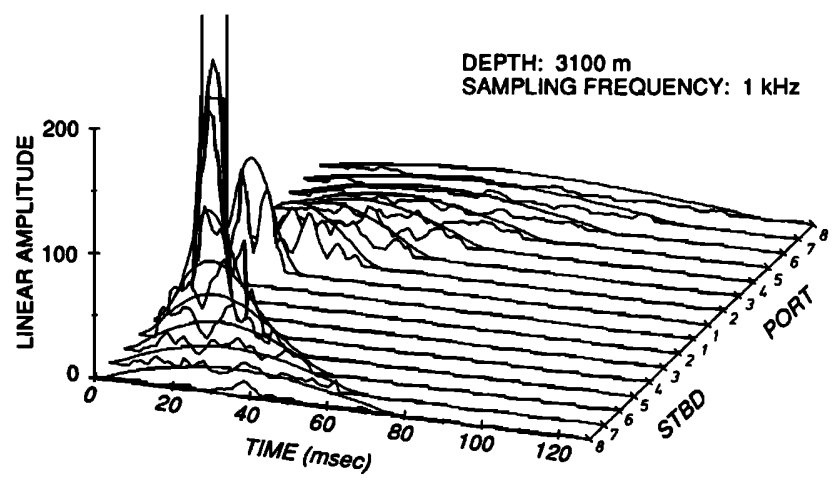

FIG. 8. Measured Sea Beam returns and their corresponding computed correction factor for one ping. The time scale is only meant to show the extent of the returns for each beam, it does not reflect the time of arrival of the echoes on those beams. Amplitudes are in relative units. 

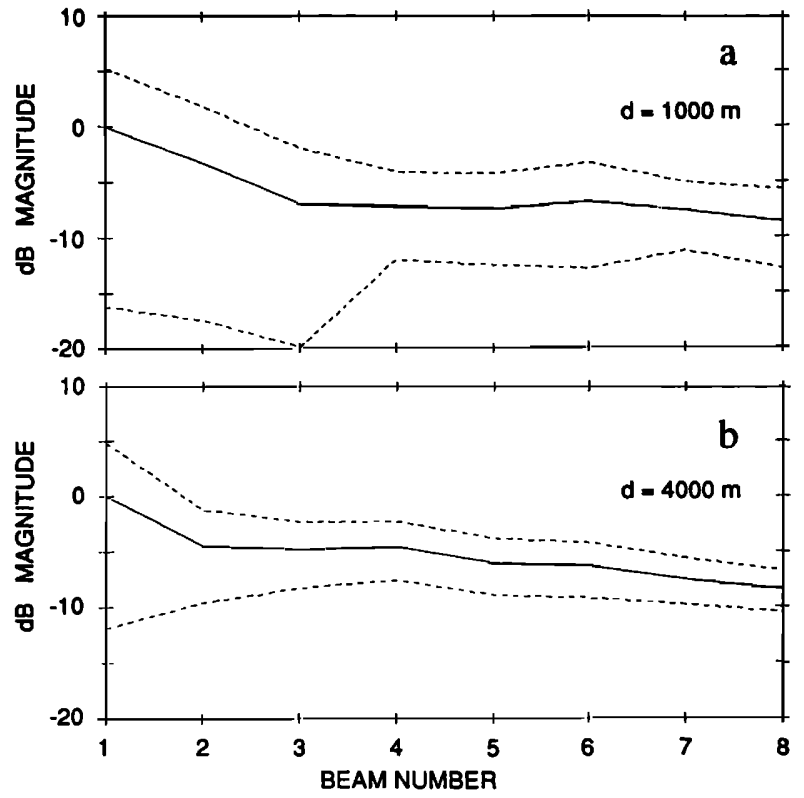

FIG. 9. Normalized apparent angular dependence of REVGEN simulated Sea Beam returns without corrections, for beams fixed in space from $0^{\circ}$ to $18.66^{\circ}$ incidence, and separated by $2.66^{\circ}$. Test cases shown are for depths of (a) $1000 \mathrm{~m}$ and (b) $4000 \mathrm{~m}$. Solid lines are the mean values and dashed lines represent 1 s.d. about the mean. The apparent angular dependence observed is only due to the beam geometry and the corresponding area insonified.

energy in the outer beams. This is presumably due to the fact that center beam returns occupy $10 \%$ or less of the window width, and their contributions are weighted down by the many small noise spikes found in the window.

\section{RESULTS AND DISCUSSION}

In the case of measured Sea Beam acoustic data, the corresponding mean energy values are binned in $1^{\circ}$ bins. This angular resolution is made possible, despite the $2.66^{\circ}$ beam separation, by the roll of the ship. The resulting angular dependence functions for two sites in the central North Pacific, one at 1500-m depth on top of Horizon Guyot (Fig. 10), and

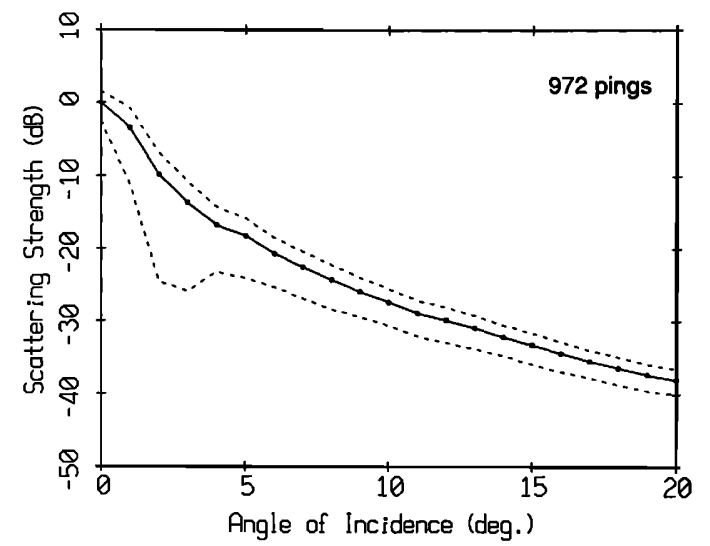

FIG. 10. Normalized angular dependence of scattering strength, with all corrections applied, for data recorded over Horizon Guyot. Dots represent the mean values in angular bins $1^{\circ}$ wide, and dashed lines represent 1 s.d. about the mean. A total of 972 pings have been binned for this plot. the other at 3100-m depth over Magellan Rise (Fig. 11) have been plotted in terms of scattering strength, normalized to its value at vertical incidence versus angle of incidence. As in the simulation results (Fig. 9), the near nadir region exhibits a large variance. In Fig. 11, the variance exceeds the mean for bins at $2^{\circ}$ and $3^{\circ}$ incidence. Although there is insufficient data to make a case, the simulation results show increased variance in the near nadir region for decreasing depth and the opposite is observed in the measured data.

Sidelobe interference effects can be ruled out, because the simulation data did not include sidelobe contributions. A more plausible explanation comes from the geometry shown in Fig. 5, and the concept of a transition region going from a beam-limited regime to a pulse-limited one. In the transition region, one expects a mix of scattering by a relatively large area bound on the side nearest vertical incidence by the width of the beam, and a growing set of returns backscattered by incremental areas sampled by the pulse propagating along the interface away from normal incidence. In the first case, contributions to the overall scattering strength for a given angular bin will sum constructively or destructvely in a random fashion from one ping to the next. By contrast, in the pulse-limited region, the propagating pulse allows for multiple measurements within each beam footprint. The result is a spatial averaging process that reduces the variance of the mean energy estimate.

Along track bottom slope variations could also produce similar effects in the near nadir region and might account for some of the increased variability observed in those data. However, although we have not addressed along track bottom slopes, it would not help explain the comparable increase in variability seen in the simulation results over the same angular sector, because the simulations did not include any slope effects.

\section{A. Comparison with theory}

In order to attempt to fit the measured data with theoretical curves based on the scattering model outlined in Sec. I, knowledge of a number of environmental parameters is

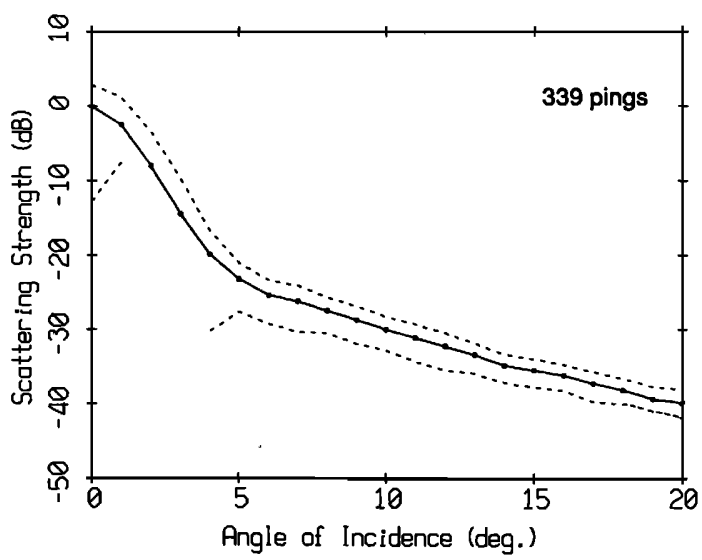

FIG. 11. Normalized angular dependence of scattering strength, with all corrections applied, for data recorded over Magellan Rise. Same binning and plot conventions as for Fig. 10, but only 339 pings were used for this plot. 
required. It would be desirable to have measurements leading to estimates of the roughness of the interface at the two sites where data were collected. Unfortunately, the horizontal length scales of the relief sampling performed by the Sea Beam system is much too sparse (point separation on the order of $5 \%$ of water depth) to be able to derive a roughness spectrum for use here. As for the density and velocity ratios at the sediment water interface, we know that both sites are covered with foraminiferal ooze with high calcium carbonate contents. ${ }^{18}$ Sediment thickness is roughly $160 \mathrm{~m}$ on Horizon Guyot and $400 \mathrm{~m}$ on Magellan Rise. Based on the above, we used a sound velocity ratio of 1.02 and a density ratio of 1.456 for the model. These values come from tables compiled by Hamilton and Bachman, ${ }^{19}$ and are used to compute the plane-wave reflection coefficient.

Because the curve fitting process involves three parameters: the two interface roughness parameters $\alpha$ and $\beta$ and the ratio $\sigma_{v} / \alpha_{b}$ controlling the sediment volume scattering contribution, we proceeded iteratively. Families of curves were derived for fixed values of $\beta$ with values of $\alpha$ varying between 1 and 0.5 . The family of curves with the closest match to the data was then examined to select a single $\alpha$ and refine the fit by including sediment volume contributions where necessary.

This procedure yielded two solutions for each data set: For the data collected over Horizon Guyot, a good fit was obtained with $\alpha=0.59, \beta=410^{-5}$ and no volume contribution in the angular sector from $5^{\circ}$ to $20^{\circ}$ incidence [Fig. 12(a) ]. On the other hand, the data in the near nadir sector from $0^{\circ}$ to $5^{\circ}$ incidence fit best with $\alpha=0.48$ and $\beta=3.510^{-5}$ [Fig. 12(b) ]. For the data collected over Magellan Rise, the angular sector from about $6^{\circ}$ to $20^{\circ}$ incidence is well matched by a curve with $\alpha=0.55, \beta=3.510^{-5}$ and no sediment volume scattering contributions [Fig. 13(a)]. A better fit is obtained with $\alpha=0.65$ and $\beta=3.510^{-5}$ for the angular sector between nadir and $6^{\circ}$ incidence [Fig. $13(b)]$.

\section{B. Discussion}

In all four cases presented in Figs. 12 and 13, no amount of volume scattering would improve the curve fitting and one is left with the choice between a curve fit favoring the near nadir region $\left(0^{\circ}-6^{\circ}\right.$ incidence) or the "outer beams" $\left(5^{\circ}-6^{\circ}\right.$ to $20^{\circ}$ incidence). Based on the beam versus pulse limitation arguments developed previously, and based on the fact that the variance of the data is worst in the near nadir region, we opted for the outer beams solution [Figs. 12(a) and 13(a) ]. The corresponding values of $\alpha(0.55$ and 0.59$)$ are within the limits of validity given in Sec. I, and are consistent with results presented by Jackson et al., ${ }^{6}$ where $\alpha=0.63$ is taken as a "generic" value. Following the assumption that interface roughness has a power spectrum given by Eq. (6), to compare results based on the roughness parameter $\beta$, we need to compute the factor $C_{h}$ of the structure function [Eqs. (7) and (8)]. For $\alpha=0.55$ and $\beta=3.5$ $10^{-5}, C_{h}=0.0203$ in a meter scale. Likewise $\alpha=0.59$ and $\beta=410^{-5}$ yield $C_{h}=0.0213$ in a meter scale. In both cases these values correspond to rms height differences $\left(C_{h} r^{\alpha}\right)$ of about $0.02 \mathrm{~m}$ for points separated by $r=1 \mathrm{~m}, 0.25-0.32 \mathrm{~m}$
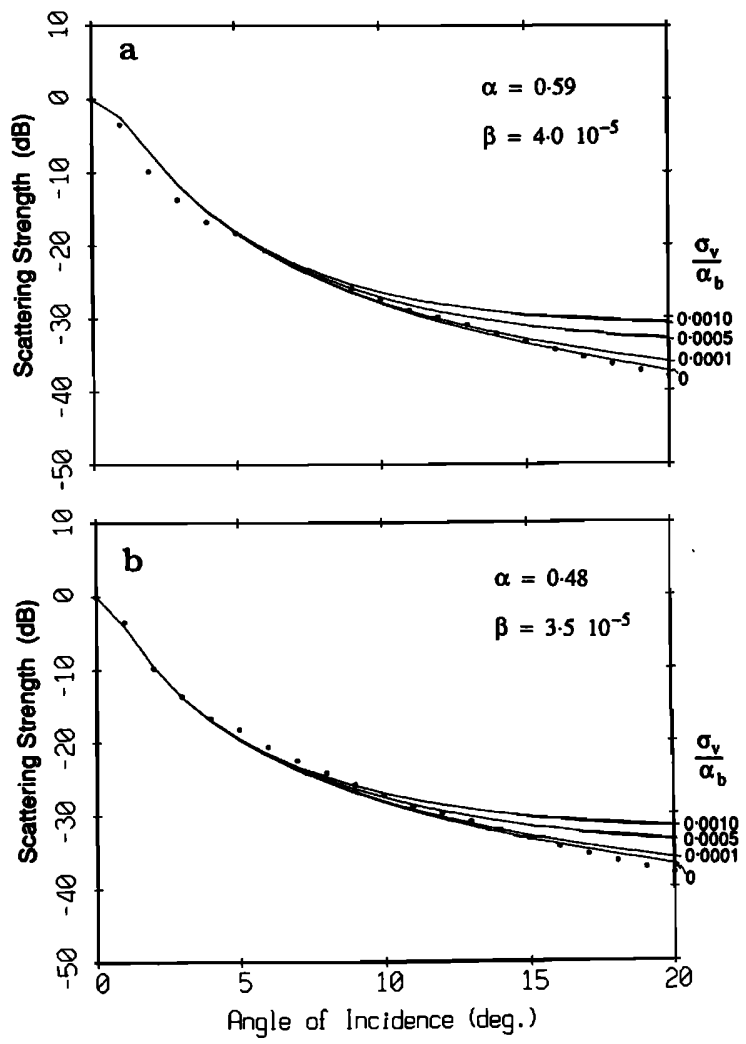

FIG. 12. Measured versus theoretical normalized scattering strength curves for the Horizon Guyot data. Theoretical curves contain both the interface scattering term Eq. (3) and the sediment volume scattering term Eq. (10) and are displayed for various values of the ratio $\sigma_{v} / \alpha_{b}$. (a) best fit in the $5^{\circ}$ to $20^{\circ}$ angular sector, (b) best fit in the $0^{\circ}$ to $5^{\circ}$ near nadir region. The fitting parameters $\alpha$ and $\beta$ are indicated on each plot. Sediment volume scattering contributions appear to be negligible in (a) and do not help the fit in (b).

for $r=100 \mathrm{~m}$, and $0.9-1.25 \mathrm{~m}$ for $r=1000 \mathrm{~m}$. This type of roughness appears quite plausible for the foraminiferal ooze sediments covering the two sites considered here. With sediment volume scattering contributions considered negligible for the chosen fit [Figs. 12(a) and 13 (a)], we infer that, for these data, surface roughness is the controlling mechanism for acoustic backscatter.

Looking beyond the simple curve fitting application presented here, and assuming that multi beam echosounders will be calibrated on a routine basis in the future, one can expect to use the corresponding absolute backscattering strength at normal incidence and the three fitting parameters as "feature vectors" for automatic seafloor classification schemes. There is not enough physical differences between the two data sets presented here to say whether bottom types can be differentiated on the basis of the angular dependence of their backscattering strength. Likewise, the "resolution" of the feature vectors will have to be validated with actual physical parameters to determine what spread of values for $\alpha$ and $\beta$ one should expect for a given substrate.

Finally, if one wishes to produce maps of seafloor scattering strength over an area, it will be necessary to first derive angular dependence functions as described here, so that all the measurements can be normalized to a given angle of 

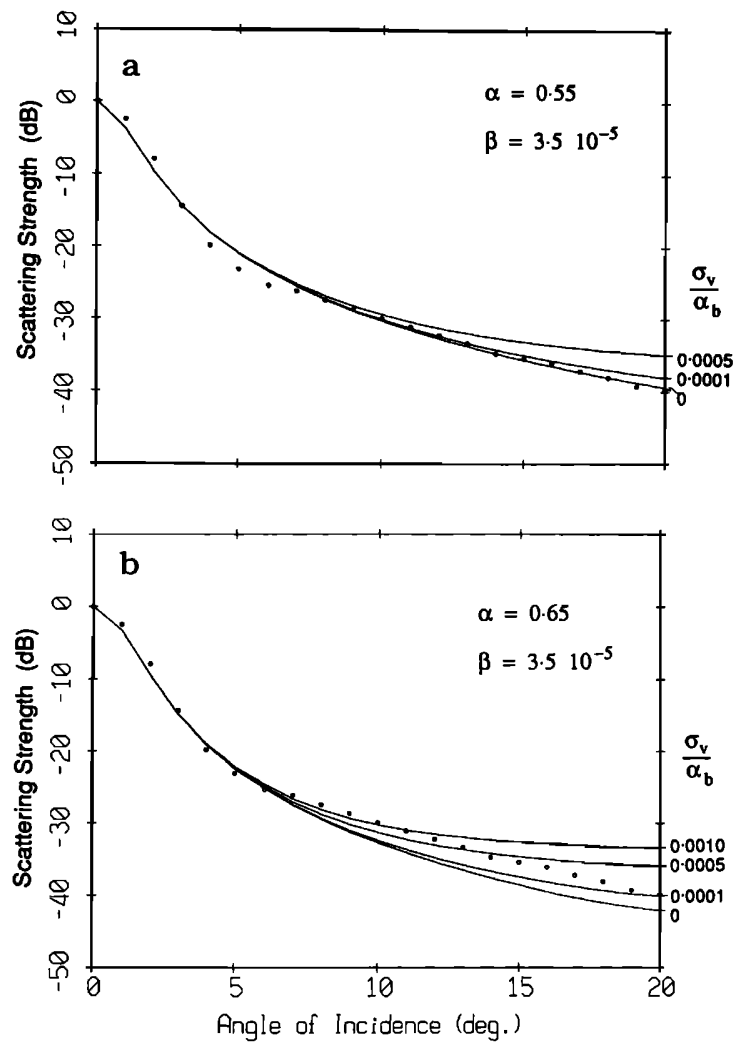

FIG. 13. Measured versus theoretical normalized scattering strength curves for the Magellan Rise data. Same representation as for Fig. 12. (a) best fit in the $6^{\circ}$ to $20^{\circ}$ angular sector, (b) best fit in the $0^{\circ}$ to $6^{\circ}$ near nadir sector. As for Fig. 12, sediment volume scattering contributions appear negligible in (a) and do not help the fit in (b). Contributions from the volume sediment scattering term are shown for various values of the ratio $\sigma_{v} / \alpha_{b}$.

incidence. The result would then be plotted in geographic coordinates, much like a bathymetric contour map, but with surface elevation replaced by bottom backscattering strength at the specified angle of incidence and acoustic frequency.

\section{CONCLUSIONS}

We have presented a comprehensive methodology for the derivation of angular dependence functions for seafloor acoustic backscatter measured with a multibeam echosounder. Three main steps were outlined: (1) sidelobe interference removal via adaptive noise cancelling techniques, (2) angular corrections to account for the ship's roll, for refraction effects through the water column and for apparent bottom slopes athwartships, and (3) geometric corrections that include effects due to the shape of the beam patterns and the size of the insonified area. Along track bottom slopes have not been addressed, but their effect will have to be included when dealing with data sets collected over rugged terrain.

Good agreement was found between the interface scattering theory, based on the Kirchhoff assumption, and measurements made with Sea Beam over an angular sector ranging from $5^{\circ}$ to $20^{\circ}$ incidence. The near nadir region (below $5^{\circ}$ incidence) was found to yield unreliable estimates of the scattering strength that exhibits a large variance in this sector. Because this behavior was also observed in simulated data, we surmise that contributions to the scattering strength in this angular sector are due to an ensemble of scatterers insonified all at once, and whose backscattered returns add constructively or destructively on any given ping. Sediment volume scattering contributions were deemed negligible in the data presented here.

\section{ACKNOWLEDGMENTS}

The authors wish to thank D. Jackson for fruitful discussions of his scattering model, B. Sotirin for review and editorial help, R. M. Lawhead for software contributions, and $\mathrm{J}$. Griffith for the art work. Funding from the Office of Naval Research through Contract No. N00014-87-K-0010 is also gratefully acknowledged.

${ }^{1}$ R. J. Urick, "The backscattering of sound from a harbor bottom," J. Acoust. Soc. Am. 26, 231-235 (1954).

${ }^{2} R$. J. Urick, "The processes of sound scattering at the ocean surface and bottom," J. Marine Res. 15, 134-148 (1956).

${ }^{3}$ C. M. McKinney and C. D. Anderson, "Measurements of backscattering of sound from the ocean bottom," J. Acoust. Soc. Am. 36, 158-163 (1964).

${ }^{4}$ H. K. Wong and W. D. Chesterman, "Bottom backscattering near grazing incidence in shallow water," J. Acoust. Soc. Am. 44, 1713-1718 (1968).

${ }^{5}$ A. V. Bunchuk and Y. Y. Zhitkovskii, "Sound scattering by the ocean bottom in shallow-water regions (review)," Sov. Phys. Acoust. 26, 363370 (1980).

${ }^{6}$ D. R. Jackson, D. P. Winebrenner, and A. Ishimaru, "Application of the composite roughness model to high-frequency bottom backscattering," J. Acoust. Soc. Am. 79, 1410-1422 (1986).

${ }^{7}$ D. R. Jackson, A. M. Baird, J. J. Crisp, and P. A. G. Thomson, "Highfrequency bottom backscatter measurements in shallow water," J. Acoust. Soc. Am. 80, 1188-1199 (1986).

${ }^{8}$ H. Boehme, N. P. Chotiros, L. D. Rolleigh, S. P. Pitt, A. L. Garcia, T. G. Goldsberry, and R. A. Lamb, "Acoustic backscattering at low grazing angles from the ocean bottom. I. Bottom backscattering strength," J. Acoust. Soc. Am. 77, 962-974 (1985).

${ }^{9} \mathrm{M}$. Gensane, "A statistical study of acoustic signals backscattered from the sea bottom," IEEE J. Oceanic Eng. 14, 84-93 (1989).

${ }^{10}$ B. C. Heezen and C. D. Hollister, The Face of the Deep (Oxford U. P., 1971).

"C. de Moustier, "Beyond bathymetry: Mapping acoustic backscattering from the deep seafloor with Sea Beam," J. Acoust. Soc. Am. 79, 316-331 (1986).

${ }^{12}$ R. P. Goddard, "REVGEN, High-fidelity simulation of sonar signals," APL-UW 8805, Applied Physics Laboratory, University of Washington, Seattle, WA (1985).

${ }^{13}$ R. J. Urick, Principles of Underwater Sound (McGraw-Hill, New York, 1983), 3rd ed., Chap. 8.

${ }^{14} \mathrm{C}$. de Moustier and F. V. Pavlicek, "A fully transportable Sea Beam complex acoustic data acquisition system," (Offshore Technology Conference, 1987).

${ }^{15} \mathrm{D}$. Alexandrou and C. de Moustier, "Adaptive noise cancelling applied to Sea Beam sidelobe interference rejection," IEEE J. Oceanic Eng. 13, 7076 (1988).

${ }^{16} \mathrm{C}$. de Moustier, "State of the art in swath bathymetry survey systems," Internat. Hyd. Rev. 65, 25-54 (1988).

${ }^{17}$ P. J. Huber, Robust Statistics (Wiley, New York, 1981).

${ }^{18}$ K. L. Smith, W. C. Schwab, M. Noble, and C. de Moustier, "Physical, geological and biological studies on four Pacific seamounts: Introduction," Deep-Sea Res. 36, 1785-1790 (1990).

${ }^{19}$ E. L. Hamilton and R. T. Bachman, "Sound velocity and related properties of marine sediments," J. Acoust. Soc. Am. 72, 1891-1904 (1982). 